\title{
Tackling antimicrobial resistance in lower urinary tract infections: treatment options
}

\author{
Johann D Pitout ${ }^{\text {abdef* }}$, Wilson Chan ${ }^{\text {ab }} \&$ Deirdre L Church $^{\text {abce }}$ \\ ${ }^{a}$ Division of Microbiology, Calgary Laboratory Services, University of Calgary, Alberta, Canada \\ ${ }^{\mathrm{b}}$ Departments of Pathology and Laboratory Medicine' University of Calgary, Alberta, Canada \\ ${ }^{\mathrm{c}}$ Medicine, University of Calgary, Alberta, Canada \\ ${ }^{\mathrm{d}}$ Microbiology, Immunology, and Infectious diseases, University of Calgary, Alberta, Canada \\ e Snyder Institute for Chronic Diseases, University of Calgary, Alberta, Canada \\ ${ }^{\mathrm{f}}$ Department of Medical Microbiology, University of Pretoria, Pretoria, South Africa.
}

\section{Abstract}

Urinary tract infections (UTIs) are among the most common infectious diseases occurring in either the community or healthcare settings. A wide variety of bacteria are responsible for causing UTIs, however extra-intestinal pathogenic E. coli or ExPEC) remains the most common etiological agent. Since 2000, resistance to antibiotics emerged globally among ExPEC and is causing delays in appropriate therapy with subsequent increased morbidity and mortality. For patients with acute uncomplicated lower UTIs, nitrofurantoin, trimethoprim-sulfamethoxazole, fosfomycin or pivmecillinam should be prescribed for a 1-5 day course depending on the agent used. Single-dose fosfomycin is an excellent option for uncomplicated lower UTIs and has had similar clinical and/or bacteriological efficacy for 3- or 7-day regimens for alternate agents (i.e., ciprofloxacin, norfloxacin, cotrimoxazole or nitrofurantoin). The aim of this review article is to provide an overview on the definitions, etiology, treatment guidelines (including agents for 
infections due to antimicrobial resistant bacteria) of lower UTIs and to highlight recent aspects on antimicrobial resistance of ExPEC.

Keywords: Lower UTIs, antimicrobial resistance, treatment, E. coli ST131

\section{Introduction}

Urinary tract infections (UTIs) are among the most common infectious diseases occurring in either the community or healthcare settings. In North America, these infections account for over 10 million physician visits annually, 1 million emergency department visits, and over 100,000 hospitalizations annually, with an estimated cost of \$1 billion per year in the U.S [1]. UTIs accounts for substantial morbidity, mortality and medical expenses on a global scale and it is estimated that approximately $20 \%$ of women over the age of $18-29$ years will suffer from an UTI during their lifetimes [2]. Moreover, UTIs are the most common bacterial infections found in nursing home residents, accounting for $50 \%$ of reported infections in Norwegian nursing homes, $30 \%$ to $50 \%$ in U.S. nursing homes, and $25 \%$ of all infections in the noninstitutionalized elderly in the U.S. Recurrent or relapsing UTIs are especially problematic in many individuals.

A wide variety of bacteria are responsible for causing UTIs, however Escherichia coli remains the most common etiological agent [1]. E. coli is an incredible versatile bacterium in that it has the ability to colonize numerous hosts (including humans) and forms an important part of the normal intestinal flora. Sometimes E. coli acquires pieces of DNA that gives it the ability to cause various diarrheal diseases and is referred to as an intestinal-pathogenic E. coli. However, there is another type of $E$. coli that has the capacity to cause infections outside the gastrointestinal system. These types of $E$. coli are referred to as extra-intestinal pathogenic $E$. 
coli or ExPEC [3]. Certain factors (referred to as virulence-associated factors) give ExPEC the means to cause infections [4]; they include toxins, adhesins, lipopolysaccharides, capsules, proteases and invasins that are encoded by genes in pathogenicity islands. ExPEC is the most common cause of UTIs in developed and developing countries [5]. Before the 2000's, ExPEC was susceptible to first line antibiotics (e.g. cephalosporins, cotrimoxazole, and fluroquinolones) that are often used to treat UTIs [5]. Unfortunately, since 2000, resistance to these antibiotics emerged globally and is causing delays in appropriate therapy with subsequent increased morbidity and mortality [5]. This has occurred at a time when the discovery and development of new anti-infective agents with activity against Gram negative bacteria are slowing down.

The aim of this review article is to provide an overview on the definitions, etiology, treatment guidelines (including agents for infections due to antimicrobial resistant bacteria) of lower UTIs and highlight recent aspects on antimicrobial resistance of ExPEC.

\section{Definitions and Etiology of lower urinary tract infections}

A UTI is defined as a bacterial infection of the urinary tract; more practically, it is the combination of significant bacteriuria and symptoms consistent with infection e.g. dysuria, frequency, fever [6]. UTIs are often further classified in dichotomies for clarity of discussion. In terms of anatomy, UTIs are divided into lower tract and upper tract infections. Lower tract UTIs, or cystitis, are infections that are limited to the bladder, whereas upper tract UTIs, or pyelonephritis, involve the kidney as well [7].

The differentiation between uncomplicated and complicated UTIs is not as clear cut, but generally uncomplicated UTIs refer to those that occur in a normal urinary tract, both anatomically and neurologically [8]. Conversely, complicated UTIs are characterized by complicating features that causes impairment of urinary flow or of host defenses. These may 
include structural abnormalities (e.g. stones, diverticula, urethral valves), functional abnormalities (e.g. neurologic impairment secondary to spinal cord injury or multiple sclerosis), presence of foreign devices (e.g. urinary catheters, ureteral stents), or an otherwise compromised host (e.g. renal transplantation, immunosuppression, pregnancy). UTIs in males are often considered complicated, as are those in diabetic patients [7]. Both uncomplicated and complicated UTI's may be lower or upper tract in location.

Ascension through the urethra is most common route of infection for the urinary tract [1]. The uropathogen is part of the rectal flora, which has a transition period of periurethral and distal urethral colonization. The bacteria, utilizing a suite of virulence factors including adhesins, fimbriae, and pili, can adhere to the uroepithelium and withstand the mechanical forces present during urination.

The most common etiologic agent of uncomplicated UTIs is Escherichia coli, implicated in $75-90 \%$ of cases [1]. Staphylococcus saprophyticus can cause a significant proportion of infections (5-15\%) in young women, but is uncommon outside this age group. Other Enterobacteriaceae, such as Klebsiella spp. and Proteus spp., are also important uropathogens. Enterococcus spp. and Streptococcus agalactiae are commonly isolated; however, these organisms are part of the periurethral flora and recent data suggests that their isolation in uncomplicated UTIs, even in high colony counts, may represent contamination [9].

Complicated UTIs have a broader microbiological range. E. coli remains a dominant uropathogen, but higher incidences of other bacteria are seen. A wider range of Enterobacteriaceae may be involved, including Klebsiella spp., the urease-producers (Proteus spp., Morganella morganii, Providencia spp.), and the other intrinsic AmpC producers (Enterobacter spp., Citrobacter spp., Serratia marcescens) [10]. Pseudomonas aeruginosa may 
be frequently involved, especially in long-term catheters. Enterococcus spp., S. agalactiae, and coagulase-negative staphylococci are the most common Gram-positive uropathogens in complicated UTI's, and likely play a more important role in this group than in uncomplicated UTIs [10]. In addition, complicating factors often predispose towards recurrences and multiple courses of antibiotics, raising the likelihood of antimicrobial-resistant organisms $[8,10]$.

\section{Antimicrobial resistance among ExPEC}

Antibiotic therapy with cotrimoxazole, cephalosporins, and fluoroquinolones are considered as the treatments of choice for infections due to ExPEC, especially before the 2000s. The development of resistance to the carbapenems among ExPEC is of special concern to the medical community at large, because these agents are often the last line of effective therapy available for the treatment of serious infections due to these bacteria [5]. Recently a new type of colistin resistant mechanism named MCR-1 was described in animals and patients in China and was associated with $E$. coli.

The most important $\beta$-lactamases in ExPEC are the extended-spectrum $\beta$-lactamases [ESBLs] (e.g. CTX-M types), plasmid-mediated or imported Amp C $\beta$-lactamases (e.g. CMY types), and to a lesser extent the carbapenemases (e.g. KPC types, MBLs e.g. VIM, IPM and NDM types, and the oxacilinases e.g. OXA-48-like enzymes) [4]. The NDM, OXA-48 and KPC are the most common carbapenemases among nosocomial and community isolates of ExPEC [11].

\section{The CTX-M $\beta$-lactamases}

Extended-spectrum $\beta$-lactamases (ESBLs) were first described in 1983 and have the ability to hydrolyse the penicillins, cephalosporins and monobactams, but not the cephamycins 
and carbapenems [12]. They are inhibited by $\beta$-lactamase inhibitors such as clavulanic acid, sulbactam and tazobactam. Although ESBLs have been identified in a range of Enterobacteriaceae, they are most often present in E. coli and K. pneumoniae. The majority of ESBLs identified in clinical isolates during the 1980s and 1990s were of the SHV or TEM types, which evolved from parent enzymes such as TEM-1, -2 and SHV-1 [12]. A different type of

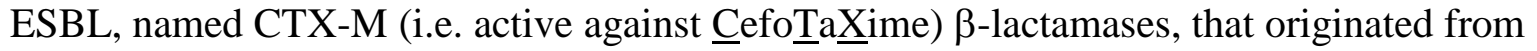
environmental Kluyvera spp, gained prominence in the 2000s with reports of clinical isolates of E. coli producing these enzymes from Europe, Africa, Asia, South and North America [13]. Since the mid 2000's CTX-M $\beta$-lactamases have also been identified in different members of the Enterobacteriaceae as well as non- Enterobacteriaceae, but remain especially common in E. coli, and has become the most wide-spread and common type of ESBL identified worldwide [14].

The CTX-Ms belong to the molecular class A or functional group $2 \beta$-lactamases, and include at least six lineages (i.e. CTX-M-1, CTX-M-2, CTX-M-8, CTX-M-9, CTX-M-25 and KLUC) that differ from each other by $\geq 10 \%$ amino acid similarity [13]. Currently, the most widespread and prevalent type of CTX-M enzyme among human clinical isolates of $E$. coli is CTX-M-15. E. coli producing this enzyme often belongs to sequence type 131 (ST131) [15]. The intercontinental dissemination of this sequence type has played a major role in the worldwide emergence of CTX-M-15 producing E. coli.

CTX-M-producing ExPEC are important causes of hospital and community-onset urinary tract infections, bacteraemia and intra-abdominal infections [13]. The risk factors associated with infections caused by CTX-M-producing E. coli include the following: repeat UTIs, underlying renal pathology, previous antibiotics including cephalosporins and fluoroquinolones, previous 
hospitalization, nursing home residents, older males and females, Diabetes Mellitus, underlying liver pathology and international travel to high risk areas such as the Indian subcontinent [16].

Global surveys have illustrated an alarming trend of associated resistance to other classes of antimicrobial agents among CTX-M-producing E. coli that include trimethoprimsulfamethoxazole, tetracycline, gentamicin, tobramycin and ciprofloxacin [14]. This has important clinical implications because the fluoroquinolones and cotrimoxazole are popular oral treatments options for UTIs. Fortunately, fosfomycin and nitrofurantoin retain sufficient activity against a high percentage of $E$. coli that produce CTX-Ms [17].

It is evident today that CTX-M-producing E. coli is a major player in the world of antimicrobial resistance. A report from the Infectious Diseases Society of America listed ESBLproducing E. coli as a priority drug-resistant microbe to which new therapies are urgently needed [18].

\section{AmpC $\beta$-lactamases}

Enterobacteriaceae with inducible cephalosporinases or AmpC $\beta$-lactamases such as Enterobacter cloacae, Citrobacter freundii, Serratia marcescens, Morganella morganii, and Providencia stuartii, can develop resistance to the oxyimino-cephalosporins and 7- $\alpha$-methoxycephalosporins (i.e. cephamycins e.g. cefoxitin, cefotetan) and monobactams (e.g. aztreonam) by overproducing their chromosomal AmpC $\beta$-lactamase [19]. In Klebsiella spp, Salmonella spp., and $P$. mirabilis, that lack chromosomal $\beta$-lactamases, this type of resistance is usually mediated by plasmid encoded or imported AmpC $\beta$-lactamases [20]. E. coli is different in that it possesses genes encoding for chromosomal non-inducible AmpC $\beta$-lactamases that are regulated by weak promoters and strong attenuators, resulting in low amounts of the cephalosporinase [21]. Surveys of resistance mechanisms in cephamycin-resistant E. coli, have identified several 
promoter or attenuator mutations, which resulted in the up-regulation and production of the natural occurring chromosomal AmpC B-lactamases [22] . Occasionally, cephamycin-resistant $E$. coli can also produce plasmid-mediated or imported AmpC $\beta$-lactamases [19].

AmpC $\beta$-lactamases at high levels, hydrolyse penicillins, most cephalosporins, cephamycins and monobactams, but not the fourth generation cephalosporins (e.g. cefepime) and carbapenems (Table 1). Resistance to the fourth generation cephalosporins are caused by point mutations in AmpC $\beta$-lactamases and is called extended-spectrum cephalosporinases. AmpC enzymes are not inhibited by "classical" $\beta$-lactamase inhibitors, such as clavulanic acid, sulbactam and tazobactam, although boronic acids and cloxacillin have shown to be good inhibitors [23]. The genes are typically encoded on large plasmids containing additional antibiotic resistance genes that are responsible for multi-resistant phenotype, leaving few therapeutic options [21].

Just like ESBL-producing bacteria, E. coli with plasmid-mediated AmpC enzymes have mostly been responsible for nosocomial outbreaks on a worldwide basis during the late 1980's and 1990's, although the risk factors associated with infection are not as well defined as those associated with ESBL-producing bacteria [21]. In a study reported from Korea, patients infected by plasmid-mediated AmpC-producing organisms had similar clinical features and outcomes to those patients infected with TEM- or SHV-related ESBL producers [24].

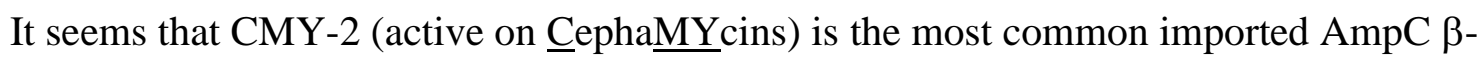
lactamase reported in Enterobacteriaceae (especially E. coli and Salmonella spp.) from different areas of the world [19]. Hospital surveys from Asia, North America and Europe have shown that the DHA types of cephamycinases are mostly present in Klebsiella spp from Asia, CMY are 
Table 1. Treatment Regimens and Expected Early Efficacy Rates for Acute Uncomplicated Cystitis*

\begin{tabular}{|c|c|c|c|c|}
\hline \multirow[b]{2}{*}{ Drug (dosage) } & \multicolumn{4}{|c|}{ Mean percentage (range) } \\
\hline & $\begin{array}{l}\text { Estimated clinical } \\
\text { efficacy }^{\mathrm{ab}}\end{array}$ & $\begin{array}{l}\text { Estimated } \\
\text { microbiological } \\
\text { efficacy }\end{array}$ & Common side effects & References \\
\hline $\begin{array}{l}\text { Nitrofurantoin monohydrate/ } \\
\text { macrocrystals ( } 100 \mathrm{mg} \text { twice daily for } \\
5-7 \text { days) }\end{array}$ & $93(84-95)$ & $88(86-92)$ & Nausea, headache & 10,21 \\
\hline $\begin{array}{l}\text { Trimethoprim-sulfamethoxazole } \\
\text { (160/800 mg twice daily for } 3 \text { days) }\end{array}$ & $93(90-100)$ & $94(91-100)$ & $\begin{array}{l}\text { Rash, urticarial, nausea, } \\
\text { vomiting, hematologic }\end{array}$ & 10,21 \\
\hline $\begin{array}{l}\text { Fosfomycin trometamol ( } 3 \mathrm{~g} \\
\text { single-dose sachet) }\end{array}$ & 91 & $80(78-83)$ & Diarrhea, nausea, headache & 10,22 \\
\hline $\begin{array}{l}\text { Pivmecillinam ( } 400 \mathrm{mg} \text { twice } \\
\text { daily for 3-7 days) }\end{array}$ & $73(55-82)$ & $79(74-84)$ & Nausea, vomiting, diarrhea & $10,30,66$ \\
\hline $\begin{array}{l}\text { Fluoroquinolones (does varies } \\
\text { by agent; 3-day regimen) }\end{array}$ & $90(85-98)$ & $91(81-98)$ & $\begin{array}{l}\text { Nausea/vomiting, } \\
\text { diarrhea, headache, } \\
\text { drowsiness, insomnia }\end{array}$ & 10,30 \\
\hline $\begin{array}{l}\beta \text {-lactams (dose varies by } \\
\text { agent; } 305 \text { day regimen) }\end{array}$ & $89(79-98)$ & $82(74-98)$ & $\begin{array}{l}\text { Diarrhea, nausea, vomiting, } \\
\text { rash, urticarial }\end{array}$ & 10,14 \\
\hline \multicolumn{5}{|c|}{$\begin{array}{l}\text { Efficacy rates refer to cure rates on the visit closest to a 5-9 day period following treatment, and are averages or ranges } \\
\text { calculated from clinical trials discussed in the text. }\end{array}$} \\
\hline \multicolumn{5}{|c|}{$\begin{array}{l}{ }^{b} \text { Estimated clinical efficacy and microbiological efficacy rates should not necessarily be compared across agents, because study } \\
\text { design, efficacy definition, therapy duration, and other factors are heterogeneous. Note that efficacy rates may vary } \\
\text { geographically depending on local patterns of antimicrobial resistance among uropathogens. }\end{array}$} \\
\hline \multicolumn{5}{|c|}{${ }^{\mathrm{c}}$ Data on fluoroquinolones are compiled from regimens of ofloxacin, norfloxacin, and ciprofloaxacin } \\
\hline $\begin{array}{l}\text { d Data on } \beta \text {-lactams are derived fro } \\
\text { clavulanate. }\end{array}$ & clinical trials exam & ining second and & rd generation cephalosporins & d amoxicillin- \\
\hline
\end{tabular}


present in E. coli from Asia, North American and Europe while FOX are present in Klebsiella spp. from North America and Europe [25-29].

\section{KPC $\beta$-lactamases}

The most clinical significant of the class A carbapenemase is the KPC-types (i.e.

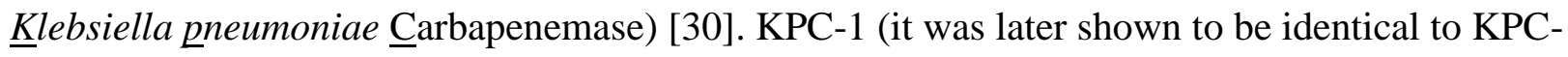
2) was reported in the late 1990's from a K. pneumoniae isolated in North Carolina, USA and to date, more than 18 different KPC variants have been described [31], even though KPC-2 and -3 remain the most commonly identified variants. These enzymes provide resistance to the carbapenems, cephalosporins, cephamycins and monobactams and are weakly inhibited by "classical” $\beta$-lactamase inhibitors such as clavulanic acid and tazobactam (Table 1). KPC $\beta$ lactamases (especially KPC-2 and -3) have been described in several enterobacterial species, especially Klebsiella spp. and to a lesser extent in Enterobacter spp. [30].

Several nosocomial outbreaks, most often due to K. pneumoniae, have been reported from North America (especially the USA), South America (Colombia, Argentina), Europe (Greece, Italy, Poland), Asia (China) and Middle East (Israel) [31-33]. KPC-producing bacteria are considered to be endemic in certain parts of the world, such as the North Eastern USA, Puerto Rico, Colombia, Greece, Italy, Israel and China and are important causes of nosocomially-acquired infections in some parts of these countries (Table 1) [33]. K. pneumoniae ST258 with KPC-2 and KPC-3 had significantly contributed to the world-wide distribution of this resistance trait [33].

The genes responsible for the production of KPC enzymes are located on transferable plasmids and are associated with the mobile element transposon $\operatorname{Tn} 4401$, explaining their spread among clinically relevant pathogens [34] (Table 1). Plasmids that encode for KPC enzymes often 
contain several genes that encode for resistance to other antimicrobial agents, such as the aminoglycosides, plasmid-mediated quinolone resistance, trimethoprim, sulfonamides and tetracyclines. This ensures that KPC-producing Enterobacteriaceae are often multiresistant to various groups of antibiotics [35].

\section{NDM $\beta$-lactamases}

A new type of metallo- $\beta$-lactamase (MBL), named NDM (i.e. New Delhi metallo- $\beta$ lactamases), was described in 2009 in K. pneumoniae and E. coli recovered from a Swedish patient who was hospitalized in New Delhi, India [36]. NDMs have the ability to hydrolyse a wide variety of $\beta$-lactams, including the penicillins, cephalosporins and carbapenems, but not the monobactams (i.e. aztreonam), and are inhibited by metal chelators such as EDTA. NDM-1 shares very little identity with other MBLs, the most similar being VIM-1/VIM-2 with only $32.4 \%$ amino acid identity. Since the first description of NDM-1, more than eight variants of this enzyme have been described, with the majority of them originating from Asia [11].

The majority of NDM-1-producing bacteria are broadly resistant to various drug classes and also carry a diversity of other resistance mechanisms (e.g. to aminoglycosides and fluoroquinolones), which leaves limited treatment options [37]. These additional mechanisms include the following: plasmid-mediated AmpC $\beta$-lactamases (especially CMY types), ESBLs (especially CTX-M-15), different carbapenemases (e.g. OXA-48-, VIM-, KPC-types), 16S RNA methylases, plasmid-mediated quinolone resistance determinants to quinolones, macrolide modifying esterases, and rifampicin-modifying enzymes. Consequently, Enterobacteriaceae with NDMs remain only susceptible to colistin, fosfomycin and tigecycline [11].

Kumarasamy and colleagues [38], provide compelling evidence that NDM-producing Enterobacteriaceae (mostly K. pneumoniae and E. coli) are widespread in India and Pakistan. 
They also found that many UK patients infected with NDM-producing bacteria had recently traveled to India to undergo several types of medical procedures. The patients presented with a variety of hospital- and community-associated infections with urinary tract infections (UTIs) being the most common clinical syndrome. Recent reports from the subcontinent (including India, Pakistan and Bangladesh) showed that the distribution of NDM $\beta$-lactamases among Enterobacteriaceae was widespread through these countries $[39,40]$ : e.g. a hospital in Varanasi in Northern India identified NDM-1 prevalence rate of 6\% among E. coli $(\mathrm{n}=528)$ from outpatients and hospitalized patients between February 2010 and July 2010 [41], $7 \%$ among E. coli from a major hospital in Mumbai, India [42], whereas 15\% (30/200) of in- and outpatients in Rawalpindi, Pakistan carried E. coli with NDM-1 in their gut flora [43]. The prevalence of asymptomatic fecal carriage was estimated to have been between 5 to $15 \%$ in these countries [11].

Since 2010 Enterobacteriaceae with NDMs have been reported worldwide from patients with an epidemiological link to the Indian subcontinent [44]. The impact of intercontinental travel as a source of spread of NDM producers has been extensively reported and these enzymes are now one of the most common carbapenemases identified in countries such as Canada, the UK and France [11]. Recent findings suggest that the Balkan states, North Africa and the Middle East might act as secondary reservoirs for the spread of NDMs, which may or may not initially have reached these countries from the Indian subcontinent [11] (Table 1).

Enterobacteriaceae (especially $K$. pneumoniae and E. coli) with NDMs, have been recovered from many clinical settings, reflecting the disease spectra of these opportunistic bacteria, including hospital and community-onset urinary tract infections, septicaemia, pulmonary infections, peritonitis, device-associated infections and soft tissue infections 
$[37,39,44]$. The frequent identification of NDMs within $E$. coli is of special concern because this organism is the most common cause of community and hospital onset urinary tract infections and diarrhea [88]. NDM-1-positive bacteria have been recovered from the gut flora of travelers returning from the Indian subcontinent and were undergoing microbiological investigation for unrelated diarrheal symptoms [45]. There is also widespread environmental contamination by NDM-1-positive bacteria in New Delhi [46].

\section{OXA-48-like $\beta$-lactamases}

The Ambler class D ß-lactamases is commonly referred to as OXAs (i.e. “oxacillinases") and comprises over 400 enzymes and some variants possess carbapenemase activity [93]. These enzymes (also referred to as carbapenem-hydrolysing class D ß-lactamases or CHDLs) do not efficiently hydrolyse the oxyimino-cephalosporins or the monobactams (the exception being OXA-163) [37]. The CHDLs possess weak carbapenemase activities that do not confer high level resistance to carbapenems if not associated to other factors such as permeability defects. The majority of CHDLs variants have been identified in Acinetobacter spp., but OXA-48 and its derivatives (i.e. OXA-163, OXA-181, OXA-204, and OXA-232) are most often encountered in Enterobacteriaceae [47].

OXA-48 was first identified in 2003 from K. pneumoniae isolated in Turkey [48] and since then, bacteria that produce these $\beta$-lactamases, have been important causes of nosocomial outbreaks in this country [49]. The first report of OXA-48-producing K. pneumoniae outside Turkey was in 2007 from Belgium [50] and bacteria that produce OXA-48 have rapidly spread to several Belgian hospitals [51]. Since then Enterobacteriaceae with OXA-48 have disseminated throughout Europe, and it seems that Turkey and North African countries (especially Morocco and Tunisia [37]) are the main reservoir for these infections. Subsequently several nosocomial 
outbreaks of OXA-48-producing K. pneumoniae, E. coli, and Enterobacter cloacae have been reported in France, Germany, Switzerland, Spain, the Netherlands and UK [47].

The spread of OXA-48 is mostly due to a mobile $62.5-\mathrm{kb}$ plasmid that belongs to the Inc L/M replicon group, as well as the presence of $\operatorname{Tn} 1999$ [47]. Interestingly, the $62.5-\mathrm{kb}$ plasmid does not carry additional resistance genes. This plasmid has the ability to efficiently transfer resistance to other enterobacterial species. OXA-48-like enzymes have mostly been identified in K. pneumoniae and E. coli to a lesser extent and are not inhibited by metal chelators such as EDTA or "classical" $\beta$-lactamases inhibitors such as clavulanic acid or tazobactam (Table 1). The production of ESBLs and/or permeability barriers in bacteria that co-produce OXA-48 will increase the level of resistance to the cephalosporins and carbapenems [37].

Several point mutant variants of OXA-48, most often in $K$. pneumoniae, have recently been reported. These includeed OXA-163, OXA-181, OXA-204 and OXA-232 [11]. OXA-163 differ from the other OXA-48-like enzymes in that its carbapenemase activity is low combined with enhanced hydrolysis of the oxyimino-cephalosporins providing isolates with resistance phenotypes similar to those bacteria with ESBL. OXA-163 was originally identified from enterobacterial isolates (E. cloacae and K. pneumoniae) recovered in Argentina, and it seems that Enterobacteriaceae with these oxacillinases are common in this South American country [11].

\section{MCR-1: plasmid-mediated colistin resistance}

MCR-1, the first transferable plasmid-mediated colistin resistance gene, was reported in ExPEC isolates from food animals, food and patients in China [52]. Since then, detection of MCR-1-positive strains has been reported in several Enterobacteriaceae worldwide. Of clinical concern is the presence of plasmids containing MCR-1in ExPEC with carbapenemases. Such 
isolates have the potential to create pandrug resistant bacteria originating mostly from the community that will nearly be impossible to treat effectively.

\section{Guidelines for treating lower urinary tract infections}

Although acute cystitis is one of the most common infections in ambulatory women, studies show that prescribing practices vary widely despite the publication of treatment guidelines by expert groups [53-55]. The Infectious Diseases Society of America (IDSA) and the European Society for Microbiology and Infectious Diseases initially published clinical practice guidelines for the treatment of acute uncomplicated cystitis in the 1990s [56]. However, in the intervening period, treatment approaches have become more complicated because of the steady rise in antimicrobial resistance amongst uropathogens, particularly E. coli [57-61], requiring the IDSA and the ESMID to update their conjoint guidelines in 2010 [62]. Although ampicillin was initially the recommended treatment of choice for uncomplicated lower UTI, this is no longer the case because of the very high prevalence of antimicrobial resistance to both amoxicillin and ampicillin worldwide [63-65]. However, other B-lactams including cefdinir, cefaclor, amoxicillin-clavulanate and cefpodoxime-proxetil in 3-7 day regimens continue to be recommended for treatment of uncomplicated cystitis when susceptibility is confirmed, and other agents cannot be used [62]. However, many of these drugs have not been well studied for the treatment of UTI, and should currently be considered alternative agents as they generally have inferior efficacy and more adverse effects on the host's normal flora (i.e., Clostridium difficile) $[62,66,67]$. The fluoroquinolones including ofloxacin, norfloxacin, ciprofloxacin and levofloxacin are also currently being considered alternative antimicrobials for acute cystitis [62]. Although fluoroquinolones are highly efficacious in a 3-day regimen, there has been a dramatic increase in resistance to these agents in Gram-negative uropathogens in the past decade, 
particularly due to the global dissemination of the ESBL-producing E. coli ST131, and Pseudomonas aeruginosa for which there are currently no other orally available treatment options[57,61,68-70]. Fluoroquinolones also have potentially serious side effects (i.e., polyneuropathothy) and an increased risk of ecological disruption of the host normal flora (i.e., C. difficile), and should therefore be reserved for more important treatments other than acute cystitis $[71,72]$.

Figure 1 and Table 1 outline the current expert recommendations for the optimal treatment for acute uncomplicated cystitis [62]. For women with acute uncomplicated cystitis who are able to take oral medication, nitrofurantoin (NTF), trimethoprim-sulfamethoxazole (SXT), fosfomycin (FOS) or pivmecillinam (PVM) should be prescribed for a 3-5 day course depending on the agent used. Nitrofurantoin monohydrate/macrocrystals is an appropriate treatment choice due to the minimal resistance [62], efficacy and propensity to cause alteration in the host's flora compared to a 3-day course of SXT [73]. Although SXT is still widely used and recommended for acute cystitis therapy, the caveat is that local resistance rates of uropathogens should not exceed $20 \%$, or if the infecting strain is confirmed to be susceptible [62]. The IDSA/ESMID chose a cut-off of $20 \%$ resistance rate for SXT based on clinical, in vitro and mathematical modelling studies with regards to the potential for treatment failure [62]. Fosfomycin-trometamol can also be used if it is available because of minimal resistance and propensity for collateral damage $[74,75]$. Although fosfomycin may be used for other indications, its use has been restricted to treatment of acute cystitis due to ESBL-producing $E$. coli or Klebsiella spp. Fosfomycin appears to have inferior efficacy however, when compared to other standard short course regimens according to data submitted to the US Food and Drug Administration (FDA)[76,77]. Several other recent studies have demonstrated comparable 
Figure 1. Recommended Approach for Selection of Appropriate Antimicrobial Therapy for Acute Uncomplicated Cystitis. DS, double-strength: UTI, urinary tract infection. Adapted with permission from IDSA/ESCMID Guidelines (10)

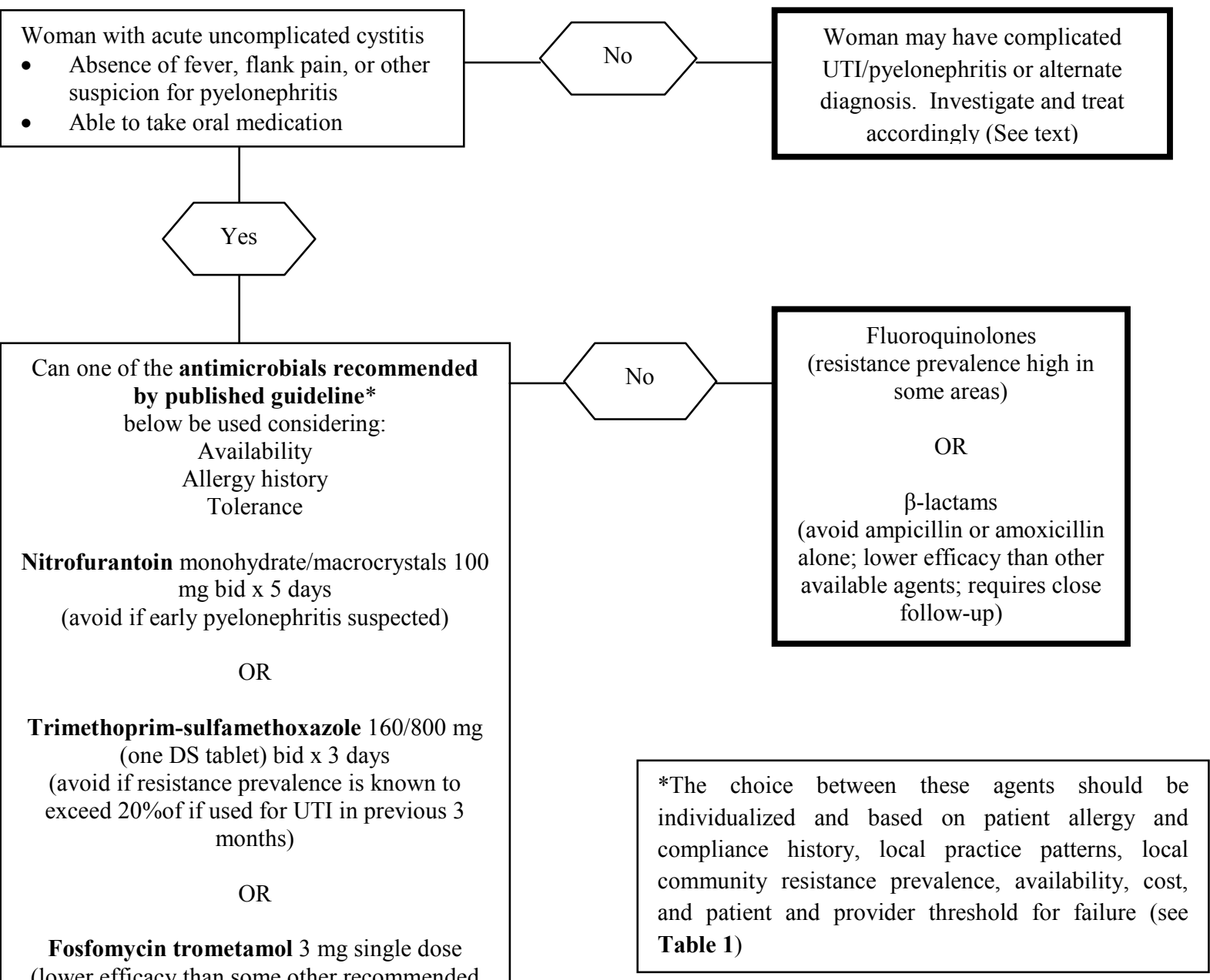
(lower efficacy than some other recommended agents; avoid if early pyelonephritis suspected)

$$
\text { OR }
$$

Pivmecillinam $400 \mathrm{mg}$ bid $\mathrm{x} 5$ days (lower efficacy than some other recommended agents; avoid if early pyelonephritis suspected)

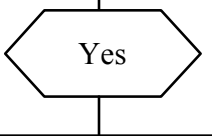

Prescribe a recommended antimicrobial 
efficacy to other regimens[78]. Finally, Pivmecillinam is an appropriate choice for therapy in some European countries where it is available. This agent is currently not licensed and/or available in North America except through special authorization access. Use of this antimicrobial for acute uncomplicated UTI in Northern European countries for several decades has resulted in minimal resistance and propensity for collateral host damage $[79,80]$. However, several recent studies have shown that Pivmecillinam may have inferior efficacy compared with other standard short course regimens, which could have been due to under dosing $[81,82]$. Women with an acute uncomplicated UTI who receive inadequate antimicrobial treatment may develop subclinical pyelonephritis with recurrent symptomatic infections with the same organism [83].

\section{Agents for treating lower urinary tract infections due to antimicrobial resistant bacteria}

The emergence of multi-drug resistant Gram-negative bacilli has renewed interest in the use of older antimicrobial agents to treat uncomplicated UTIs, particularly those that have low levels of resistance against $\beta$-lactamase-producing Enterobacteriaceae. Although nitrofurantoin and SXT have been used worldwide for decades, fosfomycin, temocillin and pivmecillinam are not licensed and/or available in North America and many countries in Europe. As such, there is a paucity of international surveillance data because currently none of these drugs are part of an antibiotic resistant surveillance in developed countries. This section describes agents that have recently become part of the new IDSA treatment recommended for acute uncomplicated UTIs, specifically fosfomycin and pivmecillinam for ExPEC, when resistance to one or more of carbapenems, colistin, aminoglycosides and quinolones are present. 


\section{Fosfomycin}

This compound has been in use for over forty years, and it has a unique mechanism of action. Fosfomycin-trometamol (FOS) is a phophonic acid derivative that acts as a bacterial cell wall inhibitor $[84,85]$. The compound inhibits the UDP-N-acetylglucosamine enolpyruvyl transferase (MurA), an enzyme responsible for ligating phosphoenolpyruvate to the 3'-hydroxyl group of UDP-N-acetylglucosamine in the first step of peptidoglycan synthesis $[77,84,86]$. The drug enters into the active site of MurA and inhibits the enzyme by covalent binding with the active site, Cys115 [87,88]. Resistance most commonly occurs by decreased permeability, and enzymatic modification of FOS by plasmid-encoded glutathione transferases (encoded by mainly fos $\mathrm{A}$, but also fos $\mathrm{B}$, fos $C$, and fos $\mathrm{X})$ [78,87-89]. Uncommonly, the fosfomycin binding site Cys115 may also be changed via mutations in the genes encoding the hexose phosphate transport (UhpT) and glycerol-3-phosphate transport (GlpT) pathways [78,87]. Resistance to FOS is rare considering the amount of time this compound has been used, due to the compound's unique structure and distinct mechanism of action $[87,88]$. However, increasing reports of plasmidmediated FOS resistance signal that this trend may not last. The plasmid-mediate fosfomycin resistance gene fos $A 3$ in $E$. coli was first described in Japan, and encodes a glutathione Stransferase[90]. Since that time, there have been increasing reports that document detection of plasmid-mediated FOS resistance (fosA3 and fos $C 2)$ in multi-drug resistant $E$. coli isolated from livestock and animals from several countries in Southeast Asia, particularly China [91-94]. More recently, plasmid-mediated FOS resistance in $E$. coli has also been reported in a patient in the United States [95].

FOS has a broad spectrum of antibacterial activity, and in vitro testing shows good activity against Gram-negative (Enterobacteriaceae) and Gram-positive bacteria 
(Staphylococcus saprophyticus, Enterococcus faecalis, but not E. faecium) that commonly cause UTIs [85,96]. A recent systematic review by Falagas et al [74], evaluated 17 selected studies of the antimicrobial activity of FOS in isolates of Enterobacteriaceae collected after 2000, and its clinical effectiveness in treating not only lower urinary tract infections and other sites (i.e., wounds). Four of these studies involved isolates from Spain [97-100], three from France [101103], two from the UK [104,105], and two from Thailand [106,107]. The remaining six studies involved isolates from Greece, Hong Kong, Japan, Korea, Turkey or the USA [108-113]. Although most of these studies used either disc diffusion or agar dilution methods, various MIC interpretive guidelines were used including: CLSI (i.e., MIC $\leq 64 \mathrm{mg} / \mathrm{L}$ ) (www.clsi.org), British Society for Antimicrobial Chemotherapy (MIC $=\leq 128 \mathrm{mg} / \mathrm{L}$ ) (www.bsac.org), and the Comité de l'Antibiogramme de la Société Française de Microbiologie (MIC $\leq 32 \mathrm{mg} / \mathrm{L}$ ) (www.sfmmicrobiologie.org)[74]. In addition, one study from France and another one from Japan did not indicate the breakpoints used. A total of 5057 isolates of Enterobacteriaceae [2205 E. coli, 764 K. pneumoniae and 73 Enterobacter spp.) were studied, including 4448 (88\%) strains that produced an ESBL. E. coli was the most susceptible to FOS of ESBL-producing Enterobacteriaceae. At least $90 \%$ of Enterobacteriaceae isolates with multi-drug resistance were susceptible to FOS using a MIC breakpoint of $\leq 64 \mathrm{mg} / \mathrm{L}$ including 1604 (96.8\%) of ESBLproducing E. coli and 608 (81.3\%) Klebsiella pneumoniae. In the 11/17 studies which used CLSI MIC interpretive criteria, the cumulative susceptibility to FOS of ESBL-producing strains was 3569/3911 (91.3\%)[74]. However, ESBL-producing Enterobacteriaceae collected from ambulatory patients had a higher rate of susceptibility to FOS by the CLSI criteria (292/297, 98.3\%) compared to those recovered from hospitalized patients (1344/1519, 88.5\%). Only 4/17 of these studies evaluated the clinical effectiveness of FOS against ESBL-producing E. coli in 
patients with various risk factors [74]. Cumulatively, treatment with FOS was associated with clinical cure in 75/80 (93.8\%) patients[74], but one of these studies reported a lower rate of microbiological clearance $(41 / 52 ; 78.8 \%)$. However, in European countries where this antimicrobial has had widespread use for several decades, susceptibility levels have recently been shown to have substantially decreased, both in strains from ambulatory and hospitalized patients [70].

More recent studies from both North America and Europe have focussed on the use of FOS for lower urinary tract infections, particularly those due to ESBL-producing Enterobacteriaceae since a single dose of FOS $3 \mathrm{~g}$ achieves a high concentration in urine[77,114], and is well tolerated, with only some nausea and diarrhoea being reported[77,96]. Keating[78] reviewed the results of clinical trials by focusing on the use of single-dose FOS treatment for patients with acute lower urinary tract infections and pregnant women with asymptomatic bacteriuria. Recent randomized trials showed that single-dose FOS had similar clinical and/or bacteriological efficacy to 3- or 7-day regimens for alternate agents (i.e., ciprofloxacin, norfloxacin, cotrimoxazole or nitrofurantoin) in women with uncomplicated UTIs. A single-dose of FOS also had similar bacteriological efficacy to a 5-day course of cefuroxime axetil or a 7-day course of amoxicillin/clavulanic acid in pregnant women with asymptomatic bacturiuria, and similar clinical and/or bacteriological efficacy to a 5-day course of cefuroxime axetil or amoxicillin/clavulanic acid, or a 3-day course of ceftibuten in pregnant women with cystitis[78].

FOS is also increasingly being used in hospitalized patients to primarily treat nosocomial UTIs, particularly for $E$. coli infection due to MDR strains, and in patients where other antimicrobials may be contraindicated due to collateral damage (i.e., $C$. difficile, patient 
allergies). A recent study also evaluated the use of FOS among inpatients at a tertiary care hospital in the United States between 2009 and 2013 [75]. The number of patients with UTIs that were treated with FOS substantially increased during this period, from none to 391 in 2013 . Of the 537 patients who received FOS for any indication, physician-diagnosed UTI accounted for $74 \%$ of prescriptions, another $10 \%$ of treatments were due to asymptomatic bacturiuria, and the remainder had other indications including fever, leukocytosis, or sepsis of uncertain etiology[75]. E. coli was the most common organism isolated from the 239 patients with UTI, and $20 \%$ of these isolates were MDR[75]. Almost all patients received a single-3g-dose of FOS, which resulted in an overall clinical success rate at $48 \mathrm{~h}$ of $74.8 \%$. However, among the 89 patients with confirmed UTIs, according to national surveillance definitions, the success rate was much higher $(89.9 \%)$. Recurrent infections occurred in only $4.3 \%$ of cases and the drug was welltolerated with only a few documented adverse events[75].

A clinical trial is also underway in Spain investigating the clinical non-inferiority of intravenous FOS $(4 \mathrm{~g} / 6 \mathrm{~h})$ compared to meropenem $(1 \mathrm{~g} / 8 \mathrm{~h})$ for treating bacteremic urinary tract infections caused by ESBL-producing E. coli [115]. This phase III randomised controlled trial which completes enrollment in 2017, will compare the clinical and microbiological efficacy 5-7 days after treatment, and the safety of these two treatment arms. If FOS is shown to have noninferior results to the use of a carbapenem, the results may have justified the use of parenteral FOS as an alternate agent for treatment of selected patients with frequent complicated UTIs caused by MDR E. coli with susceptibility to this drug.

\section{Pivmecillinam}

This compound is the pro-drug of mecillinam (6 6 -amidinopenicillanic acid), which is a penicillin derivative that has been used extensively in Scandinavian countries since the 1980s for 
treatment of uncomplicated UTIs [116,117]. Mecillinam disrupts cell wall synthesis by inhibiting penicillin-binding protein 2 (PBP2)[118,119], which is responsible for elongation of rod-shaped cells. The mechanism(s) of resistance to mecillinam are currently poorly understood although a recent study showed that mutation in $c y s B$ preventing the production cysteine was found in clinical isolates. The reduced cysteine levels led to an increased cellular concentration of the ppGpp molecule, which makes the drug's PBP2 target nonessential[120].

Pivmecillinam (PVM) treatment causes minimal disruption to the gastrointestinal microbial flora, and the drug achieves high concentrations in urine [116,117,121]. PVM only has in vitro activity against Gram-negative organisms, but its extensive use for uncomplicated UTI has shown that it is safe during pregnancy, and effective for Staphylococcus saprophyticus infection because the high urine levels overcome in vitro resistance $[79,122]$. Although the Swedish Reference Group for Antibiotics (www.srga.org) has published breakpoints for the susceptibility testing of PVM against uropathogens, a recent study suggested that these should be revised [122]. Monsen et al. [122] recently analyzed data from a prospective, multi-centre, placebo-controlled primary healthcare facility in Sweden of 1143 women with cystitis who were enrolled from 1995-98. The clinical and bacteriological outcomes of PVM treatment were evaluated in this cohort with a specific focus on patients with UTI due to a PVM resistant uropathogen. In vitro susceptibility to PVM did not predict clinical or bacteriological outcome of treatment irrespective of the type of uropathogen causing infection.

To date there have been limited reports of the treatment efficacy of PVM for UTIs. Recent studies however have shown albeit in a small number of patients the PVM has bacteriologic and clinical efficacy for treatment of lower UTIs caused by ESBL-producing Enterobacteriaceae. Nicolle et al.[82] compared 3-days of PVM $400 \mathrm{mg}$ po bid or norfloxacin 
$400 \mathrm{mg}$ bid treatment of acute uncomplicated urinary infection in women. Bacteriological cure was achieved in $75 \%$ of PVM patients compared to $91 \%$ of those who received norfloxacin. Early post-therapy clinical cure was achieved in $82 \%$ of women who received PVM compared to 88\% who received norfloxacin. Although adverse effects were similar for both drugs, norfloxacin was superior for short-course therapy in terms of bacteriological cure, but clinical outcomes were less marked. More recently, Nicolle et al. [123] described the successful treatment of a patient with relapsing pyelonephritis due to an CTX-M-producing E. coli who was cured with a prolonged course of PVM. Titelman et al. [81] recently reported the efficacy of PVM for treatment of lower urinary tract infection caused by ESBL-producing E. coli and Klebsiella pneumoniae in eight patients. All patients had a good clinical response, but bacteriological cure rates were low $(2 / 8 ; 25 \%)$. However, despite persisting bacteriuria with the same uropathogen, all of the patients remained asymptomatic for 6 months after treatment. The clinical and bacteriological effect of PVM for ESBL-producing E. coli and K. pneumoniae in UTI was also reported in a larger group of 39 patients who had PVM susceptible isolates [124]. Of the 39 patients enrolled, 30 received a treatment regimen of $400 \mathrm{mg}$ of PVM TID, and the other 9 received $200 \mathrm{mg}$ TID. The overall bacteriological cure rate was $79 \%$ and was similar whether the patients received the higher $(80 \%)$ or lower (78\%) antibiotic dosage. Clinical symptoms resolved in $84 \%$ of the evaluable patients, and only five patients had relapsing bacteriuria.

\section{Expert commentary}

Urinary tract infections (UTIs) are among the most common infectious diseases occurring in either the community or healthcare settings and E. coli (extra-intestinal pathogenic E. coli or ExPEC) remains the most common etiological agent. During the 1990's, ExPEC was susceptible 
to first line antibiotics (e.g. cephalosporins, cotrimoxazole, and fluroquinolones) that were used to empirically treat UTIs. Resistance to these antibiotics emerged globally and is now causing delays in appropriate therapy with subsequent increased morbidity and mortality. The most important $\beta$-lactamases in ExPEC are the extended-spectrum $\beta$-lactamases [ESBLs] (e.g. CTXM types), plasmid-mediated or imported Amp C $\beta$-lactamases (e.g. CMY types), and to a lesser extent the carbapenemases (e.g. KPC types, MBLs e.g. VIM, IPM and NDM types, and the oxacilinases e.g. OXA-48-like enzymes). MCR-1, the first transferable plasmid-mediated colistin resistance gene, was reported in ExPEC isolates from food animals, food and patients in China.

The Infectious Diseases Society of America (IDSA) and the European Society for Microbiology and Infectious Diseases initially published clinical practice guidelines for the treatment of acute uncomplicated cystitis in the 1990s. However, in the intervening period, treatment approaches have become more complicated because of the steady rise in antimicrobial resistance amongst uropathogens, particularly E. coli, requiring the IDSA and the ESMID to update their conjoint guidelines in 2010. For women with acute uncomplicated cystitis who are able to take oral medication, nitrofurantoin, trimethoprim-sulfamethoxazole, fosfomycin or pivmecillinam should be prescribed for a 1-5 day course depending on the agent used. Singledose fosfomycin is an excellent option for uncomplicated lower UTIs and had similar clinical and/or bacteriological efficacy to 3- or 7-day regimens for alternate agents (i.e., ciprofloxacin, norfloxacin, cotrimoxazole or nitrofurantoin) in women with uncomplicated UTIs.

Pivmecillinam is available in certain North European countries and is currently not licensed and/or available in North America. Several recent studies have shown that Pivmecillinam may have inferior efficacy compared with other standard short course regimens. 


\section{Five Year View}

ExPEC will continue to be the most common cause of uncomplicated UTIs on a worldwide basis. Resistance to different classes of antibiotics will continue to increase among global ExPEC due to the expansion of successful drug resistant clones such as E. coli ST131and ST405. This will cause further delays in appropriate therapy of community and hospital onset UTIs with subsequent increased morbidity and mortality. The carbapenemases (e.g. NDMs, KPC and OXA-48), plasmid-mediated resistance to fosfomycin and colistin will most likely expand exponentially among ExPEC in the next few years or so.

The medical community urgently needs effective oral drugs for the treatment of uncomplicated UTIs. Unfortunately, the development of such new anti-infective agents will most likely not be available within the next 5 years.

\section{Funding}

This work was supported in part by a research grant from Calgary Laboratory Services (\#10009392)

\section{Key issues}

- Urinary tract infections (UTIs) are among the most common infectious diseases occurring in either the community or healthcare settings.

- A wide variety of bacteria are responsible for causing UTIs, however Escherichia coli remains the most common etiological agent.

- Before the 2000's, ExPEC was susceptible to first line antibiotics (e.g. cephalosporins, cotrimoxazole, and fluroquinolones) that are often used to treat UTIs. Unfortunately since 
2000 , resistance to these antibiotics had emerged globally and is causing delays in appropriate therapy with subsequent increased morbidity and mortality.

- The most important $\beta$-lactamases in ExPEC are the extended-spectrum $\beta$-lactamases

[ESBLs] (e.g. CTX-M types), plasmid-mediated or imported Amp C $\beta$-lactamases (e.g. CMY types), and to a lesser extent the carbapenemases (e.g. KPC types, MBLs e.g. VIM, IPM and NDM types, and the oxacilinases e.g. OXA-48-like enzymes).

- For women with acute uncomplicated cystitis who are able to take oral medication, nitrofurantoin, trimethoprim-sulfamethoxazole (SXT), fosfomycin or pivmecillinam should be prescribed for a 1-5 day course depending on the agent used.

- Single-dose fosfomycin had similar clinical and/or bacteriological efficacy to 3- or 7-day regimens for alternate agents (i.e., ciprofloxacin, norfloxacin, cotrimoxazole or nitrofurantoin) in women with uncomplicated UTIs.

- Pivmecillinam is available in certain North European countries and is currently not licensed and/or available in North America. Several recent studies have shown that Pivmecillinam may have inferior efficacy when compared to other standard short course regimens.

\section{References}

1. Nicolle LE. Urinary tract infection. Crit Care Clin, 29(3), 699-715 (2013).

2. Foxman B. The epidemiology of urinary tract infection. Nat Rev Urol, 7(12), 653-660 (2010). 
3. Riley LW. Pandemic lineages of extraintestinal pathogenic Escherichia coli. Clin Microbiol Infect, 20(5), 380-390 (2014).

4. Pitout JD. Extraintestinal Pathogenic Escherichia coli: A Combination of Virulence with Antibiotic Resistance. Frontiers in microbiology, 3, 9 (2012).

5. Pitout JD. Extraintestinal pathogenic Escherichia coli: an update on antimicrobial resistance, laboratory diagnosis and treatment. Expert review of anti-infective therapy, 10(10), 1165-1176 (2012).

6. Nicolle LE, Bradley S, Colgan R et al. Infectious Diseases Society of America guidelines for the diagnosis and treatment of asymptomatic bacteriuria in adults. Clin Infect Dis, 40(5), 643-654 (2005).

7. Wang A, Nizran P, Malone MA, Riley T. Urinary tract infections. Prim Care, 40(3), 687706 (2013).

8. Pallett A, Hand K. Complicated urinary tract infections: practical solutions for the treatment of multiresistant Gram-negative bacteria. J Antimicrob Chemother, 65 Suppl 3, iii25-33 (2010).

9. Hooton TM, Roberts PL, Cox ME, Stapleton AE. Voided midstream urine culture and acute cystitis in premenopausal women. N Engl J Med, 369(20), 1883-1891 (2013). 
10. Levison ME, Kaye D. Treatment of complicated urinary tract infections with an emphasis on drug-resistant gram-negative uropathogens. Curr Infect Dis Rep, 15(2), 109-115 (2013).

11. Nordmann P, Poirel L. The difficult-to-control spread of carbapenemase producers in Enterobacteriaceae worldwide. Clinical microbiology and infection : the official publication of the European Society of Clinical Microbiology and Infectious Diseases, (2014).

12. Paterson DL, Bonomo RA. Extended-spectrum beta-lactamases: a clinical update. Clinical microbiology reviews, 18(4), 657-686 (2005).

13. Canton R, Coque TM. The CTX-M beta-lactamase pandemic. Curr Opin Microbiol, 9(5), 466-475 (2006).

14. Pitout JD, Laupland KB. Extended-spectrum beta-lactamase-producing Enterobacteriaceae: an emerging public-health concern. The Lancet infectious diseases, 8(3), 159-166 (2008).

15. Peirano G, Pitout JD. Molecular epidemiology of Escherichia coli producing CTX-M beta-lactamases: the worldwide emergence of clone ST131 O25:H4. International journal of antimicrobial agents, 35(4), 316-321 (2010). 
16. Rodriguez-Bano J, Pascual A. Clinical significance of extended-spectrum betalactamases. Expert review of anti-infective therapy, 6(5), 671-683 (2008).

17. D'Andrea MM, Arena F, Pallecchi L, Rossolini GM. CTX-M-type beta-lactamases: a successful story of antibiotic resistance. International journal of medical microbiology : IJMM, 303(6-7), 305-317 (2013).

18. Talbot GH, Bradley J, Edwards JE, Jr., Gilbert D, Scheld M, Bartlett JG. Bad bugs need drugs: an update on the development pipeline from the Antimicrobial Availability Task Force of the Infectious Diseases Society of America. Clin Infect Dis, 42(5), 657-668 (2006).

19. Jacoby GA. AmpC beta-lactamases. Clinical microbiology reviews, 22(1), 161-182, (2009).

20. Hanson ND. AmpC beta-lactamases: what do we need to know for the future? $J$ Antimicrob Chemother, 52(1), 2-4 (2003).

21. Philippon A, Arlet G, Jacoby GA. Plasmid-determined AmpC-type beta-lactamases. Antimicrob Agents Chemother, 46(1), 1-11 (2002).

22. Forward KR, Willey BM, Low DE et al. Molecular mechanisms of cefoxitin resistance in Escherichia coli from the Toronto area hospitals. Diagn Microbiol Infect Dis, 41(1-2), $57-63$ (2001). 
23. Doi Y, Paterson DL. Detection of plasmid-mediated class C beta-lactamases. Int J Infect Dis, 11(3), 191-197 (2007).

24. Pai H, Kang CI, Byeon JH et al. Epidemiology and clinical features of bloodstream infections caused by AmpC-type-beta-lactamase-producing Klebsiella pneumoniae. Antimicrobial agents and chemotherapy, 48(10), 3720-3728 (2004).

25. Alvarez M, Tran JH, Chow N, Jacoby GA. Epidemiology of conjugative plasmidmediated AmpC beta-lactamases in the United States. Antimicrobial agents and chemotherapy, 48(2), 533-537 (2004).

26. Li Y, Li Q, Du Y et al. Prevalence of plasmid-mediated AmpC beta-lactamases in a Chinese university hospital from 2003 to 2005: first report of CMY-2-Type AmpC betalactamase resistance in China. J Clin Microbiol, 46(4), 1317-1321 (2008).

27. Moland ES, Hanson ND, Black JA, Hossain A, Song W, Thomson KS. Prevalence of newer beta-lactamases in gram-negative clinical isolates collected in the United States from 2001 to 2002. Journal of clinical microbiology, 44(9), 3318-3324 (2006).

28. Mulvey MR, Bryce E, Boyd DA et al. Molecular characterization of cefoxitin-resistant Escherichia coli from Canadian hospitals. Antimicrobial agents and chemotherapy, 49(1), 358-365 (2005). 
29. Woodford N, Reddy S, Fagan EJ et al. Wide geographic spread of diverse acquired AmpC beta-lactamases among Escherichia coli and Klebsiella spp. in the UK and Ireland. J Antimicrob Chemother, 59(1), 102-105 (2007).

30. Queenan AM, Bush K. Carbapenemases: the versatile beta-lactamases. Clin Microbiol Rev, 20(3), 440-458, (2007).

31. Walther-Rasmussen J, Hoiby N. Class A carbapenemases. J Antimicrob Chemother, 60(3), 470-482 (2007).

32. Deshpande LM, Rhomberg PR, Sader HS, Jones RN. Emergence of serine carbapenemases (KPC and SME) among clinical strains of Enterobacteriaceae isolated in the United States Medical Centers: report from the MYSTIC Program (1999-2005). Diagn Microbiol Infect Dis, 56(4), 367-372 (2006).

33. Nordmann P, Cuzon G, Naas T. The real threat of Klebsiella pneumoniae carbapenemase-producing bacteria. Lancet Infect Dis, 9(4), 228-236 (2009).

34. Naas T, Cuzon G, Villegas MV, Lartigue MF, Quinn JP, Nordmann P. Genetic structures at the origin of acquisition of the beta-lactamase bla KPC gene. Antimicrob Agents Chemother, 52(4), 1257-1263 (2008).

35. Poirel L, Pitout JD, Nordmann P. Carbapenemases: molecular diversity and clinical consequences. Future Microbiol, 2(5), 501-512 (2007). 
36. Yong D, Toleman MA, Giske CG et al. Characterization of a new metallo-beta-lactamase gene, bla(NDM-1), and a novel erythromycin esterase gene carried on a unique genetic structure in Klebsiella pneumoniae sequence type 14 from India. Antimicrob Agents Chemother, 53(12), 5046-5054 (2009).

37. Nordmann P, Naas T, Poirel L. Global spread of Carbapenemase-producing Enterobacteriaceae. Emerging infectious diseases, 17(10), 1791-1798 (2011).

38. Kamarasamy K TM, Walsh TR. Emergence of a new antibiotic resistance in India, Pakistan and the UK: end of conventional antibiotics? . Lancet Infectious Diseases (2010).

39. Castanheira M, Deshpande LM, Mathai D, Bell JM, Jones RN, Mendes RE. Early dissemination of NDM-1- and OXA-181-producing Enterobacteriaceae in Indian hospitals: report from the SENTRY Antimicrobial Surveillance Program, 2006-2007. Antimicrob Agents Chemother, 55(3), 1274-1278 (2011).

40. Lascols C, Hackel M, Marshall SH et al. Increasing prevalence and dissemination of NDM-1 metallo-beta-lactamase in India: data from the SMART study (2009). The Journal of antimicrobial chemotherapy, 66(9), 1992-1997 (2011).

41. Seema K, Ranjan Sen M, Upadhyay S, Bhattacharjee A. Dissemination of the New Delhi metallo-beta-lactamase-1 (NDM-1) among Enterobacteriaceae in a tertiary referral hospital in north India. J Antimicrob Chemother, 66(7), 1646-1647 (2011). 
42. Deshpande P, Rodrigues C, Shetty A, Kapadia F, Hedge A, Soman R. New Delhi Metallo-beta lactamase (NDM-1) in Enterobacteriaceae: treatment options with carbapenems compromised. J Assoc Physicians India, 58, 147-149 (2010).

43. Perry JD, Naqvi SH, Mirza IA et al. Prevalence of faecal carriage of Enterobacteriaceae with NDM-1 carbapenemase at military hospitals in Pakistan, and evaluation of two chromogenic media. J Antimicrob Chemother, 66(10), 2288-2294 (2011).

44. Nordmann P, Poirel L, Walsh TR, Livermore DM. The emerging NDM carbapenemases. Trends Microbiol, (2011).

45. Leverstein-Van Hall MA, Stuart JC, Voets GM, Versteeg D, Tersmette T, Fluit AC. Global spread of New Delhi metallo-beta-lactamase 1. The Lancet infectious diseases, 10(12), 830-831 (2010).

46. Walsh TR, Weeks J, Livermore DM, Toleman MA. Dissemination of NDM-1 positive bacteria in the New Delhi environment and its implications for human health: an environmental point prevalence study. The Lancet infectious diseases, 11(5), 355-362 (2011).

47. Poirel L, Bonnin RA, Nordmann P. Genetic features of the widespread plasmid coding for the carbapenemase OXA-48. Antimicrob Agents Chemother, 56(1), 559-562 (2012). 
48. Poirel L, Heritier C, Tolun V, Nordmann P. Emergence of oxacillinase-mediated resistance to imipenem in Klebsiella pneumoniae. Antimicrob Agents Chemother, 48(1), 15-22 (2004).

49. Carrer A, Poirel L, Yilmaz M et al. Spread of OXA-48-encoding plasmid in Turkey and beyond. Antimicrob Agents Chemother, 54(3), 1369-1373 (2010).

50. Cuzon G, Naas T, Bogaerts P, Glupczynski Y, Huang TD, Nordmann P. Plasmidencoded carbapenem-hydrolyzing beta-lactamase OXA-48 in an imipenem-susceptible Klebsiella pneumoniae strain from Belgium. Antimicrob Agents Chemother, 52(9), 34633464 (2008).

51. Glupczynski Y, Huang TD, Bouchahrouf W et al. Rapid emergence and spread of OXA48-producing carbapenem-resistant Enterobacteriaceae isolates in Belgian hospitals. Int $J$ Antimicrob Agents, 39(2), 168-172 (2012).

52. Liu YY, Wang Y, Walsh TR et al. Emergence of plasmid-mediated colistin resistance mechanism MCR-1 in animals and human beings in China: a microbiological and molecular biological study. Lancet Infect Dis, 16(2), 161-168 (2016).

53. Philips H, Huibers L, Holm Hansen E et al. Guidelines adherence to lower urinary tract infection treatment in out-of-hours primary care in European countries. Qual Prim Care, 22(4), 221-231 (2014). 
54. Taur Y, Smith MA. Adherence to the Infectious Diseases Society of America guidelines in the treatment of uncomplicated urinary tract infection. Clin Infect Dis, 44(6), 769-774 (2007).

55. Cai T, Verze P, Brugnolli A et al. Adherence to European Association of Urology Guidelines on Prophylactic Antibiotics: An Important Step in Antimicrobial Stewardship. Eur Urol, (2015).

56. Warren JW, Abrutyn E, Hebel JR, Johnson JR, Schaeffer AJ, Stamm WE. Guidelines for antimicrobial treatment of uncomplicated acute bacterial cystitis and acute pyelonephritis in women. Infectious Diseases Society of America (IDSA). Clin Infect Dis, 29(4), 745758 (1999).

57. Pitout JD. Infections with extended-spectrum beta-lactamase-producing enterobacteriaceae: changing epidemiology and drug treatment choices. Drugs, 70(3), 313-333 (2010).

58. Wagenlehner FM, Wagenlehner C, Naber KG, Weidner W. Current anti-infective treatment of bacterial urinary tract infections. Mini Rev Med Chem, 8(8), 790-795 (2008).

59. Giamarellou H, Poulakou G. Multidrug-resistant Gram-negative infections: what are the treatment options? Drugs, 69(14), 1879-1901 (2009). 
60. McIsaac WJ, Moineddin R, Meaney C, Mazzulli T. Antibiotic-resistant Escherichia coli in women with acute cystitis in Canada. Can J Infect Dis Med Microbiol, 24(3), 143-149 (2013).

61. Ironmonger D, Edeghere O, Bains A, Loy R, Woodford N, Hawkey PM. Surveillance of antibiotic susceptibility of urinary tract pathogens for a population of 5.6 million over 4 years. J Antimicrob Chemother, 70(6), 1744-1750 (2015).

62. Gupta K, Hooton TM, Naber KG et al. International clinical practice guidelines for the treatment of acute uncomplicated cystitis and pyelonephritis in women: A 2010 update by the Infectious Diseases Society of America and the European Society for Microbiology and Infectious Diseases. Clin Infect Dis, 52(5), e103-120 (2011).

63. Kahlmeter G, Ahman J, Matuschek E. Antimicrobial Resistance of Escherichia coli Causing Uncomplicated Urinary Tract Infections: A European Update for 2014 and Comparison with 2000 and 2008. Infect Dis Ther, 4(4), 417-423 (2015).

64. Naber KG, Schito G, Botto H, Palou J, Mazzei T. Surveillance study in Europe and Brazil on clinical aspects and Antimicrobial Resistance Epidemiology in Females with Cystitis (ARESC): implications for empiric therapy. Eur Urol, 54(5), 1164-1175 (2008).

65. Ahmed MN, Vannoy D, Frederick A, Chang S, Lawler E. First-Line Antimicrobial Resistance Patterns of Escherichia coli in Children With Urinary Tract Infection in 
Emergency Department and Primary Care Clinics. Clin Pediatr (Phila), 55(1), 19-28 (2016).

66. Hooton TM, Scholes D, Gupta K, Stapleton AE, Roberts PL, Stamm WE. Amoxicillinclavulanate vs ciprofloxacin for the treatment of uncomplicated cystitis in women: a randomized trial. JAMA, 293(8), 949-955 (2005).

67. Rodriguez-Bano J, Alcala JC, Cisneros JM et al. Community infections caused by extended-spectrum beta-lactamase-producing Escherichia coli. Arch Intern Med, 168(17), 1897-1902 (2008).

68. Hooton TM, Besser R, Foxman B, Fritsche TR, Nicolle LE. Acute uncomplicated cystitis in an era of increasing antibiotic resistance: a proposed approach to empirical therapy. Clin Infect Dis, 39(1), 75-80 (2004).

69. Zhanel GG, Hisanaga TL, Laing NM et al. Antibiotic resistance in outpatient urinary isolates: final results from the North American Urinary Tract Infection Collaborative Alliance (NAUTICA). Int J Antimicrob Agents, 26(5), 380-388 (2005).

70. Sorlozano A, Jimenez-Pacheco A, de Dios Luna Del Castillo J et al. Evolution of the resistance to antibiotics of bacteria involved in urinary tract infections: a 7-year surveillance study. Am J Infect Control, 42(10), 1033-1038 (2014). 
71. Marino K, Parlee A, Orlando R, Lerner L, Strymish J, Gupta K. Comparative Effectiveness of Single versus Combination Antibiotic Prophylaxis for Infections after Transrectal Prostate Biopsy. Antimicrob Agents Chemother, 59(12), 7273-7275 (2015).

72. Gordon D, Young LR, Reddy S, Bergman C, Young JD. Incidence of Clostridium difficile infection in patients receiving high-risk antibiotics with or without a proton pump inhibitor. J Hosp Infect, 92(2), 173-177 (2016).

73. Iravani A, Klimberg I, Briefer C, Munera C, Kowalsky SF, Echols RM. A trial comparing low-dose, short-course ciprofloxacin and standard 7 day therapy with cotrimoxazole or nitrofurantoin in the treatment of uncomplicated urinary tract infection. $J$ Antimicrob Chemother, 43 Suppl A, 67-75 (1999).

74. Falagas ME, Kastoris AC, Kapaskelis AM, Karageorgopoulos DE. Fosfomycin for the treatment of multidrug-resistant, including extended-spectrum beta-lactamase producing, Enterobacteriaceae infections: a systematic review. Lancet Infect Dis, 10(1), 43-50 (2010).

75. Sastry S, Clarke LG, Alrowais H, Querry AM, Shutt KA, Doi Y. Clinical Appraisal of Fosfomycin in the Era of Antimicrobial Resistance. Antimicrob Agents Chemother, 59(12), 7355-7361 (2015).

76. Fosfomycin for urinary tract infections. Med Lett Drugs Ther, 39(1005), 66-68 (1997). 
77. Patel SS, Balfour JA, Bryson HM. Fosfomycin tromethamine. A review of its antibacterial activity, pharmacokinetic properties and therapeutic efficacy as a singledose oral treatment for acute uncomplicated lower urinary tract infections. Drugs, 53(4), 637-656 (1997).

78. Keating GM. Fosfomycin trometamol: a review of its use as a single-dose oral treatment for patients with acute lower urinary tract infections and pregnant women with asymptomatic bacteriuria. Drugs, 73(17), 1951-1966 (2013).

79. Nicolle LE. Pivmecillinam in the treatment of urinary tract infections. J Antimicrob Chemother, 46 Suppl 1, 35-39; discussion 63-35 (2000).

80. Nicolle LE. Pivmecillinam for the treatment of acute uncomplicated urinary infection. Int J Clin Pract, 53(8), 612-617 (1999).

81. Titelman E, Iversen A, Kalin M, Giske CG. Efficacy of pivmecillinam for treatment of lower urinary tract infection caused by extended-spectrum beta-lactamase-producing Escherichia coli and Klebsiella pneumoniae. Microb Drug Resist, 18(2), 189-192 (2012).

82. Nicolle LE, Madsen KS, Debeeck GO et al. Three days of pivmecillinam or norfloxacin for treatment of acute uncomplicated urinary infection in women. Scand J Infect Dis, 34(7), 487-492 (2002). 
83. Church DL. Can a Silent Kidney Infection or Genetic Predisposition Underlie Recurrent UTIs? Medscape Womens Health, 1(9), 6 (1996).

84. Kahan FM, Kahan JS, Cassidy PJ, Kropp H. The mechanism of action of fosfomycin (phosphonomycin). Ann N Y Acad Sci, 235(0), 364-386 (1974).

85. Pogue JM, Marchaim D, Kaye D, Kaye KS. Revisiting "older" antimicrobials in the era of multidrug resistance. Pharmacotherapy, 31(9), 912-921 (2011).

86. Karageorgopoulos DE, Wang R, Yu XH, Falagas ME. Fosfomycin: evaluation of the published evidence on the emergence of antimicrobial resistance in Gram-negative pathogens. J Antimicrob Chemother, 67(2), 255-268 (2012).

87. Karageorgopoulos DE, Miriagou V, Tzouvelekis LS, Spyridopoulou K, Daikos GL. Emergence of resistance to fosfomycin used as adjunct therapy in KPC Klebsiella pneumoniae bacteraemia: report of three cases. J Antimicrob Chemother, 67(11), 27772779 (2012).

88. Ho PL, Chan J, Lo WU et al. Prevalence and molecular epidemiology of plasmidmediated fosfomycin resistance genes among blood and urinary Escherichia coli isolates. J Med Microbiol, 62(Pt 11), 1707-1713 (2013).

89. Castenada-garcia ABJ, Rodriquez-Rojas A. Molecular mechanisms and clinical impact of acquired and intrinsic fosfomycin resistance. Antibiotics, 2, 217-236. (2013). 
90. Wachino J, Yamane K, Suzuki S, Kimura K, Arakawa Y. Prevalence of fosfomycin resistance among CTX-M-producing Escherichia coli clinical isolates in Japan and identification of novel plasmid-mediated fosfomycin-modifying enzymes. Antimicrob Agents Chemother, 54(7), 3061-3064 (2010).

91. Hou J, Yang X, Zeng Z et al. Detection of the plasmid-encoded fosfomycin resistance gene fosA3 in Escherichia coli of food-animal origin. J Antimicrob Chemother, 68(4), 766-770 (2013).

92. Ho PL, Chan J, Lo WU et al. Dissemination of plasmid-mediated fosfomycin resistance fosA3 among multidrug-resistant Escherichia coli from livestock and other animals. $J$ Appl Microbiol, 114(3), 695-702 (2013).

93. Lee SY, Park YJ, Yu JK et al. Prevalence of acquired fosfomycin resistance among extended-spectrum beta-lactamase-producing Escherichia coli and Klebsiella pneumoniae clinical isolates in Korea and IS26-composite transposon surrounding fosA3. $J$ Antimicrob Chemother, 67(12), 2843-2847 (2012).

94. Hou J, Huang X, Deng Y et al. Dissemination of the fosfomycin resistance gene fosA3 with CTX-M beta-lactamase genes and rmtB carried on IncFII plasmids among Escherichia coli isolates from pets in China. Antimicrob Agents Chemother, 56(4), 21352138 (2012). 
95. Alrowais H, McElheny CL, Spychala CN et al. Fosfomycin Resistance in Escherichia coli, Pennsylvania, USA. Emerg Infect Dis, 21(11), 2045-2047 (2015).

96. Garau J. Other antimicrobials of interest in the era of extended-spectrum beta-lactamases: fosfomycin, nitrofurantoin and tigecycline. Clin Microbiol Infect, 14 Suppl 1, 198-202 (2008).

97. Andreu A, Planells I, Grupo Cooperativo Espanol para el Estudio de la Sensibilidad Antimicrobiana de los Patogenos U. [Etiology of community-acquired lower urinary infections and antimicrobial resistance of Escherichia coli: a national surveillance study]. Med Clin (Barc), 130(13), 481-486 (2008).

98. de Cueto M, Lopez L, Hernandez JR, Morillo C, Pascual A. In vitro activity of fosfomycin against extended-spectrum-beta-lactamase-producing Escherichia coli and Klebsiella pneumoniae: comparison of susceptibility testing procedures. Antimicrob Agents Chemother, 50(1), 368-370 (2006).

99. Ena J, Arjona F, Martinez-Peinado C, Lopez-Perezagua Mdel M, Amador C. Epidemiology of urinary tract infections caused by extended-spectrum beta-lactamaseproducing Escherichia coli. Urology, 68(6), 1169-1174 (2006).

100. Goyanes MJ, Cercenado E, Insa R, Morente A, Alcala L, Bouza E. High rates of antimicrobial co-resistance among Enterobacteriaceae: comparative analysis between 
clinical isolates resistant and susceptible to third-generation cephalosporins. Rev Esp Quimioter, 20(2), 216-221 (2007).

101. Dubois V, Arpin C, Noury P, Andre C, Coulange L, Quentin C. Prolonged outbreak of infection due to TEM-21-producing strains of Pseudomonas aeruginosa and enterobacteria in a nursing home. J Clin Microbiol, 43(8), 4129-4138 (2005).

102. Gouby A, Neuwirth C, Bourg G et al. Epidemiological study by pulsed-field gel electrophoresis of an outbreak of extended-spectrum beta-lactamase-producing Klebsiella pneumoniae in a geriatric hospital. J Clin Microbiol, 32(2), 301-305 (1994).

103. Arpin C, Coze C, Rogues AM, Gachie JP, Bebear C, Quentin C. Epidemiological study of an outbreak due to multidrug-resistant Enterobacter aerogenes in a medical intensive care unit. J Clin Microbiol, 34(9), 2163-2169 (1996).

104. Ellington MJ, Livermore DM, Pitt TL, Hall LM, Woodford N. Mutators among CTX-M beta-lactamase-producing Escherichia coli and risk for the emergence of fosfomycin resistance. J Antimicrob Chemother, 58(4), 848-852 (2006).

105. Woodford N, Ward ME, Kaufmann ME et al. Community and hospital spread of Escherichia coli producing CTX-M extended-spectrum beta-lactamases in the UK. $J$ Antimicrob Chemother, 54(4), 735-743 (2004). 
106. Tharavichitkul P, Kantawa B, Cousoung V, Boonchoo M. Activity of Fosfomycin against extended-spectrum B-lactamase-producing strains of Pseudomonas aeruginosa and enterobacteria in a nursing home. J infet Dis Antimicrob Agents, 22, 121-126. (2005).

107. Waiwarawooth J, K Jutiworakul K, Joraka W. The prevalence and susceptibility patterns of ESBL-producing Klebsiella pneumoniae and Escherichia coli in Chonburi Hospital. J Infect Dis Antimicrob Agents, 23, 57-65. (2006).

108. Prakash V, Lewis JS, 2nd, Herrera ML, Wickes BL, Jorgensen JH. Oral and parenteral therapeutic options for outpatient urinary infections caused by enterobacteriaceae producing CTX-M extended-spectrum beta-lactamases. Antimicrob Agents Chemother, 53(3), 1278-1280 (2009).

109. Ko KS, Suh JY, Peck KR et al. In vitro activity of fosfomycin against ciprofloxacinresistant or extended-spectrum beta-lactamase-producing Escherichia coli isolated from urine and blood. Diagn Microbiol Infect Dis, 58(1), 111-115 (2007).

110. Ho PL, Wong RC, Yip KS et al. Antimicrobial resistance in Escherichia coli outpatient urinary isolates from women: emerging multidrug resistance phenotypes. Diagn Microbiol Infect Dis, 59(4), 439-445 (2007).

111. Muratani T, Matsumoto T. Urinary tract infection caused by fluoroquinolone- and cephem-resistant Enterobacteriaceae. Int J Antimicrob Agents, 28 Suppl 1, S10-13 (2006). 
112. Pullukcu H, Aydemir S, Iskigoz Tafibakan M, Cilli F, Tunger A, Ullusoy S. Susceptibility of extended-speetrum beta-lactamase-producing Escherichia coli urine isolates fo fosfomycin, ciprofloxacin, amikacin and trimethoprim-sulfamethoxazole. Turkish J Med Sci, 38, 175-180. (2008).

113. Falagas ME, Kanellopoulou MD, Karageorgopoulos DE et al. Antimicrobial susceptibility of multidrug-resistant Gram negative bacteria to fosfomycin. Eur J Clin Microbiol Infect Dis, 27(6), 439-443 (2008).

114. Gismondo MR, Romeo MA, Lo Bue AM, Chisari G, Nicoletti G. Microbiological basis for the use of fosfomycin trometamol as single-dose therapy for simple cystitis. Chemioterapia, 5(4), 278-282 (1986).

115. Rosso-Fernandez C, Sojo-Dorado J, Barriga A et al. Fosfomycin versus meropenem in bacteraemic urinary tract infections caused by extended-spectrum beta-lactamaseproducing Escherichia coli (FOREST): study protocol for an investigator-driven randomised controlled trial. BMJ Open, 5(3), e007363 (2015).

116. Graninger W. Pivmecillinam--therapy of choice for lower urinary tract infection. Int J Antimicrob Agents, 22 Suppl 2, $73-78$ (2003).

117. Giske CG. Contemporary resistance trends and mechanisms for the old antibiotics colistin, temocillin, fosfomycin, mecillinam and nitrofurantoin. Clin Microbiol Infect, 21(10), 899-905 (2015). 
118. Spratt BG. Distinct penicillin binding proteins involved in the division, elongation, and shape of Escherichia coli K12. Proc Natl Acad Sci U S A, 72(8), 2999-3003 (1975).

119. Spratt BG. The mechanism of action of mecillinam. J Antimicrob Chemother, 3 Suppl B, 13-19 (1977).

120. Thulin E, Sundqvist M, Andersson DI. Amdinocillin (Mecillinam) resistance mutations in clinical isolates and laboratory-selected mutants of Escherichia coli. Antimicrob Agents Chemother, 59(3), 1718-1727 (2015).

121. Kerrn MB, Frimodt-Moller N, Espersen F. Urinary concentrations and urine ex-vivo effect of mecillinam and sulphamethizole. Clin Microbiol Infect, 10(1), 54-61 (2004).

122. Monsen TJ, Holm SE, Ferry BM, Ferry SA. Mecillinam resistance and outcome of pivmecillinam treatment in uncomplicated lower urinary tract infection in women. APMIS, 122(4), 317-323 (2014).

123. Nicolle LE, Mulvey MR. Successful treatment of ctx-m ESBL producing Escherichia coli relapsing pyelonephritis with long term pivmecillinam. Scand J Infect Dis, 39(8), 748749 (2007).

124. Jansaker F, Frimodt-Moller N, Sjogren I, Dahl Knudsen J. Clinical and bacteriological effects of pivmecillinam for ESBL-producing Escherichia coli or Klebsiella pneumoniae in urinary tract infections. J Antimicrob Chemother, 69(3), 769-772 (2014). 


\section{Highlighted References}

Reference 1: Excellent overview on UTIs

Reference 62: excellent guidelines from USA and Europe for treating lower UTIs.

Reference 113: good overview on fosfomycin

Reference 116: good overview on pivmecillinam 